\title{
Response Gene to Complement-32 mediates aortic smooth muscle cells differentiation in human atherosclerotic lesions
}

S. I. Vlaicu ${ }^{1,2}$, A. Tatomir ${ }^{2}$, D. Boodhoo², V. Rus ${ }^{3}$, H. Rus ${ }^{2,4}$

${ }^{1}$ Department of Internal Medicine, "Iuliu Haţieganu" University of Medicine and Pharmacy of Cluj-Napoca, Romania, ${ }^{2}$ Department of Neurology, University of Maryland School of Medicine, Baltimore MD, USA, ${ }^{3}$ Department of Internal Medicine, University of Maryland School of Medicine, Baltimore MD, USA, ${ }^{4}$ Veterans Administration, Maryland Health Care System, Baltimore, MD, USA

IntroduCtion
The shift from the differentiated contractile smooth muscle
cell (SMC) phenotype to a less differentiated, proliferative
state and excessive extracellular matrix (ECM) production,
both TGF- $\beta$ regulated events, are key alterations in
atherogenesis. The complement system takes part in the
initiation and progression of atherosclerosis. First
described as a cell cycle regulator, Response Gene to
Complement (RGC)-32 is also implicated in tumorigenesis,
immune system regulation, scar tissue formation,
regulation of lipid and glucose metabolism and
atherogenesis. We have shown that RGC-32 modulates
C5b-9-induced endothelial cell (EC) proliferation and
migration and is involved in EC cytoskeletal organization
and cell adhesion. $\underline{2}$ Additionally, RGC-32 promotes vascular
SMC proliferation. RGC-32 also mediates some of TGF- $\beta$ -
induced profibrotic effects in a variety of tissues. 5

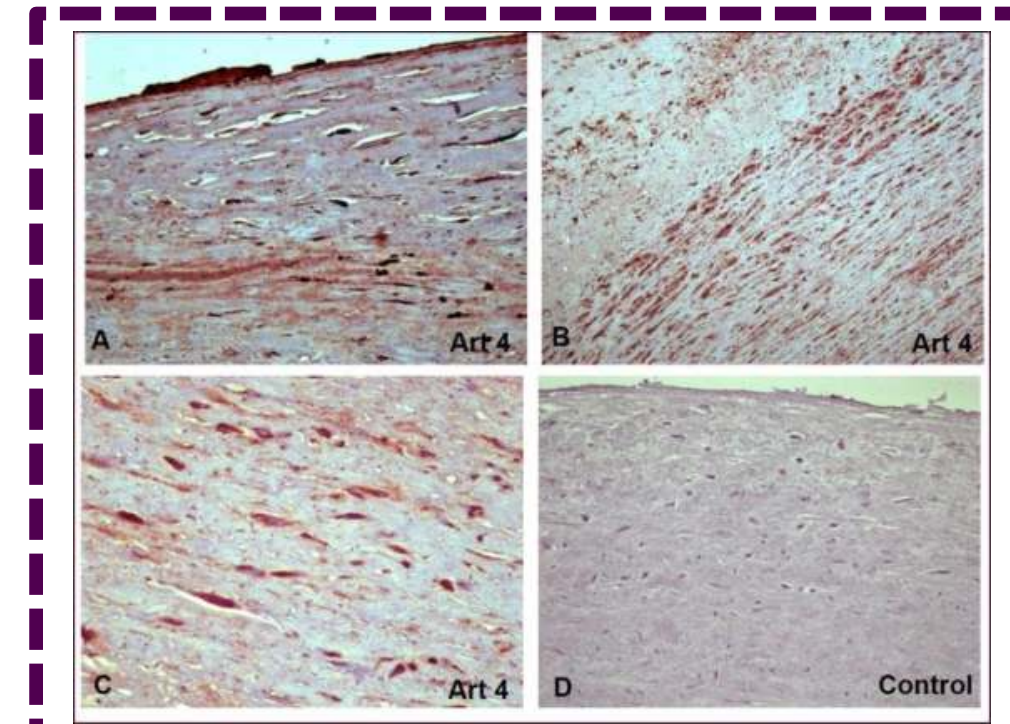

C Art $D$ Contro

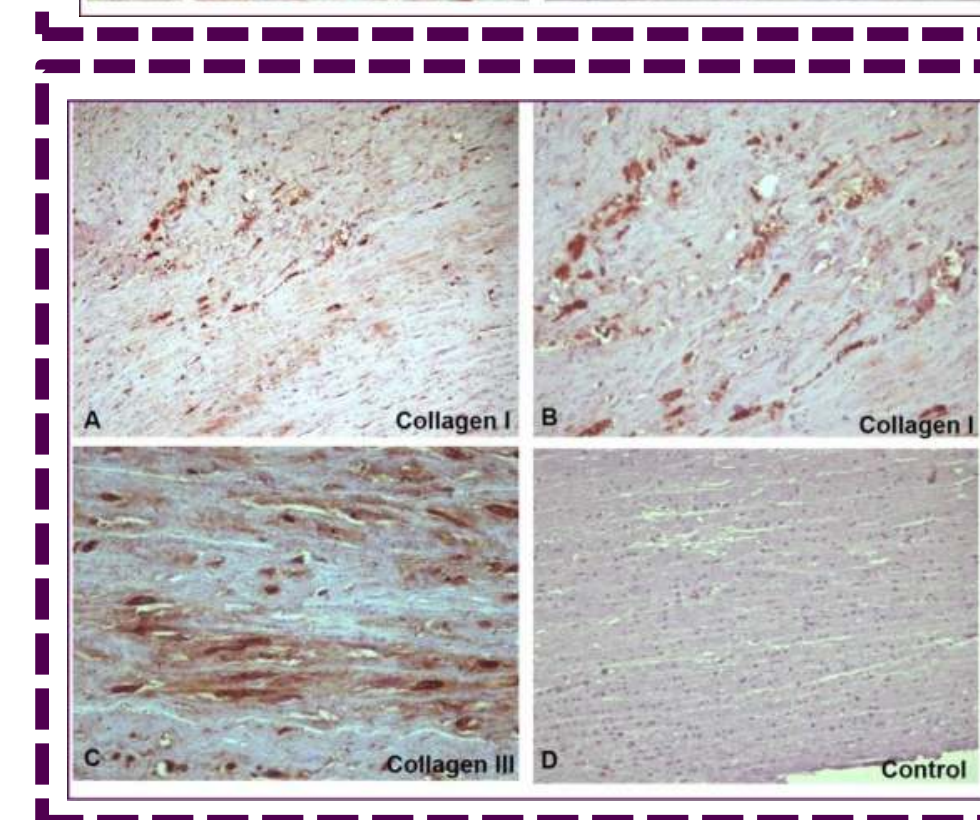

| cin collagen III

Figure 2. Immunohistochemical localization of RGC-32 in human fibrous plaques.

RGC-32 was found to be expressed by endothelial cells (A), monocytes I infiltrating the intima (A, B), ASMC I migrated in the intima and ASMC in the media (C). Controls of immunoperoxidase reaction were negative (D).

\section{Figure 3. Immunohistochemical} localization of collagen I and III in human fibrous plaques.

Collagen I and III were found to be expressed by endothelial cells (A), monocytes infiltrating the I intima (A, B), ASMC migrated in the intima $(A, B)$ and $A S M C$ in the media (C). Controls of immunoperoxidase reaction were negative (D).

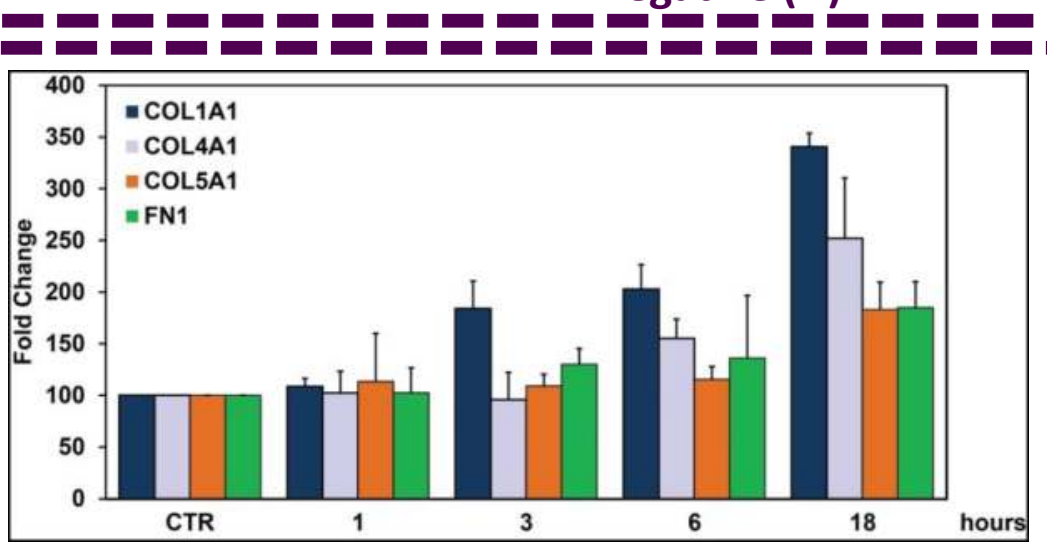
I I We examined the expression of RGC-32, alpha-smooth muscle actin ( $\alpha$-SMA - as a marker of aortic SMC differentiation), collagen type I to $\mathrm{V}$ and fibronectin (as I ECM components) in 9 human aortic atherosclerotic lesions (5 fibrous plaques and 4 intimal thickenings) by means of the indirect immunoperoxidase method. Rabbit IgG antiRGC-32 (Bioss Inc), rabbit IgG anti-collagen I, III, IV and V I (Santa Cruz Biotech) and rabbit polyclonal alpha smooth muscle Actin antibody (Abcam) along with the RTU Vecastain kit (Vector Labs) were used. Human aortic SMC (ASMC) from Lonza (Walkersville, MD) were grown 3-5 I passages in SMC basal medium, containing supplements of I $5 \% \mathrm{FBS}, 10 \mathrm{ng} / \mathrm{ml}$ human EGF, $2 \mathrm{ng} / \mathrm{ml}$ human FGF and 5 $\mu \mathrm{g} / \mathrm{ml}$ insulin. ASMC were then exposed to TGF- $\beta$ $(10 \mathrm{ng} / \mathrm{ml})$ for $1,3,6$ or 18 hours. Expression of fibronectin, pro-collagen type I, IV, V and $\alpha$-SMA was determined by I real-time PCR. The impact of RGC-32 in mediating ECM expression in ASMC was then explored via silencing RGC-32 expression in ASMC, which were transfected with siRGC-32 (Santa Cruz Biotech) or siCTR using Lipofectamine 2000. After $48 \mathrm{~h}$, cells were stimulated with TGF-ß $(10 \mathrm{ng} / \mathrm{ml})$ and total mRNA was purified and analyzed for the mRNA expression of pro-collagen I, IV, V, fibronectin and $\alpha$-SMA.

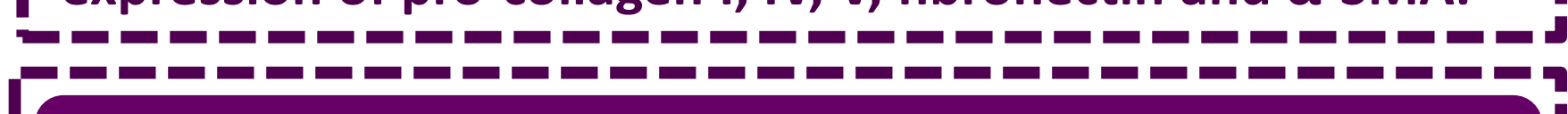

\section{Results}

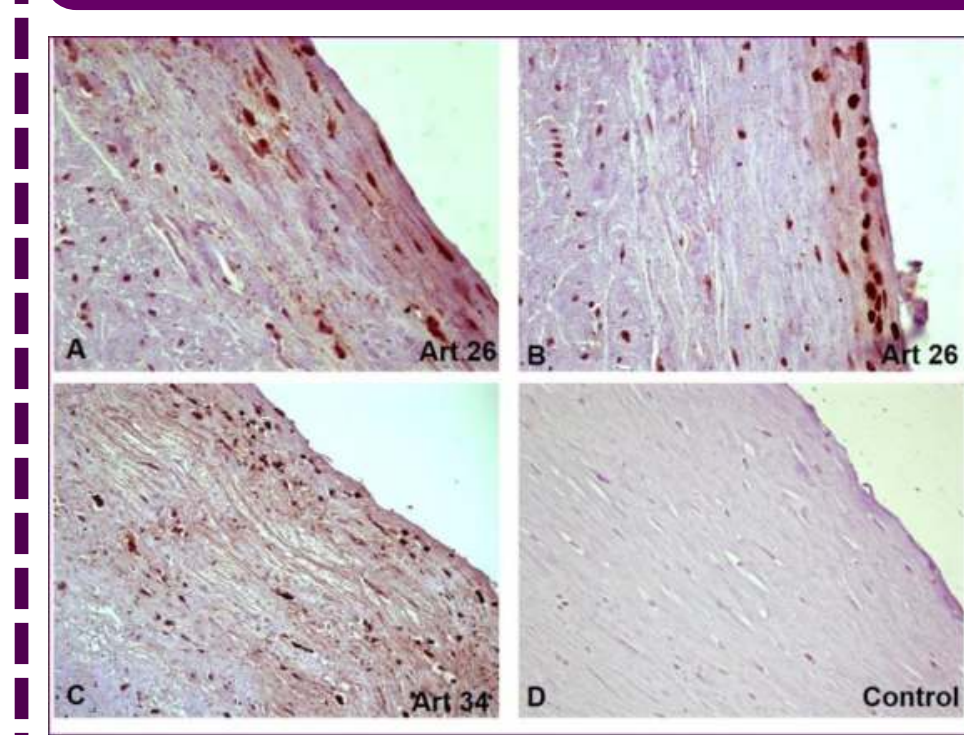

Figure 1. Immunohistochemical localization of RGC-32 in human intimal thickenings.

RGC-32 was found to be expressed by endothelial cells $(A)$, monocytes infiltrating the intima $(A, B)$ and less by smooth muscle cells in the media (C). Controls of immunoperoxidase reaction were negative (D) 32 silencing significantly reduced the mRNA expression of pro-collagens I

Figure 6. Effect of RGC-32 silencing on $\alpha$-smooth muscle actin. RGC-32 silencing in ASMC led to a significant reduction in TGF- $\beta$ induced $\alpha-S M A(p<0.02)$.

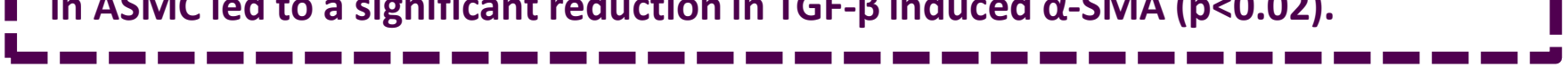

Figure 5. Effect of RGC-32 silencing on pro-collagen I, IV, V and fibronectin. RGC( $p<0.02)$, IV $(p<0.03), V$ and fibronectin $(p<0.05)(A)$ when compared with siCTR.
Our data infers that RGC-32 is a vital player in TGF- $\beta$-mediated differentiation and ECM production by ASMC, rendering it an I attractive target for therapeutic intervention in atherosclerosis.

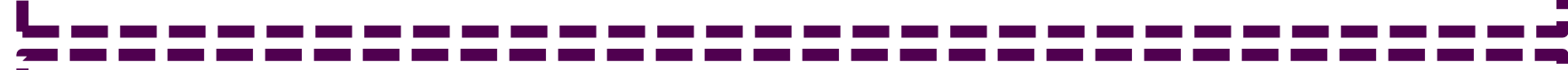
REFERENCES: 